\title{
Is Handedness Associated with the Depth of the Olfactory Sulcus?
}

\author{
Yunpeng Zang a, b Katie L. Whitcroft ${ }^{a, c, d}$ Carmina Glöckler ${ }^{a}$ \\ Thomas Hummel ${ }^{a}$ \\ a Smell and Taste Clinic, Department of Otorhinolaryngology, Medical Faculty \\ Carl Gustav Carus, TU Dresden, Dresden, Germany; ${ }^{b}$ Department of Otorhinolaryngology, \\ the Affiliated Hospital Xuzhou Medical University, Xuzhou, China; ' UCL Ear Institute, \\ London, UK; ${ }^{d}$ Centre for the Study of the Senses, Institute of Philosophy, School of \\ Advanced Studies, London, UK
}

\author{
Keywords \\ Olfactory sulcus · Handedness · Sniffin' Sticks test · Lateralization
}

\begin{abstract}
Background: Structural and functional asymmetry is frequent in biological systems. The aim of the present study was to examine whether there is a relation between handedness and the depth of the olfactory sulcus (OS) and reinvestigate whether there is a lateralization of OS depth. Methods: Forty-two healthy volunteers (mean age 24.1 years; 23 right-handed and 19 left-handed) participated. The subjects' brains were scanned using magnetic resonance imaging to assess OS depth. Normal olfactory function was ascertained using the Sniffin' Sticks odor identification test. Results: There was no significant difference in OS depth between right- and left-handed participants (right-handed mean: right OS depth $=11.3 \mathrm{~mm}$, left OS = $10.6 \mathrm{~mm}$; left-handed mean: right $\mathrm{OS}=11.3 \mathrm{~mm}$, left $\mathrm{OS}=11.0 \mathrm{~mm}$ ). Across all subjects, OS depth was significantly larger on the right side compared to the left. Conclusion: Handedness has no major influence on OS depth. The present study confirmed that the right OS is deeper compared to the left OS, possibly indicating a right-sided lateralization of the olfactory system. Implications: The depth of the right OS is larger than that of the left OS. No major effect of handedness was observed.

(c) 2020 S. Karger AG, Basel
\end{abstract}

Y. Zang and K.L. Whitcroft contributed equally to this work. 
Zang et al.: Olfactory Sulcus and Handedness

\section{Introduction}

Structural and functional asymmetry is frequent in biological systems. In humans and other mammals, such asymmetry can be demonstrated within the central nervous system. For example, more than $90 \%$ of people are right-handed, the motor control for which is located in the left hemisphere. Language processing is also lateralized, with left hemispheric dominance being demonstrated in over $95 \%$ of right-handed people. However, language lateralization is not always dependent on handedness, with left-sided dominance found in only $70 \%$ of left-handed people [1]. It is unclear whether central olfactory processing is lateralized, and, if so, to what degree this lateralization is related to handedness.

Anatomically, the peripheral olfactory system consists of two olfactory nerves that extend from the right or left nasal cavity to the corresponding ipsilateral olfactory bulb. From here, signals are transmitted along the olfactory tract, to areas of the primary and secondary olfactory networks, including the piriform cortex, amygdala and orbitofrontal cortex [2]. Some functional neuroimaging studies demonstrated higher levels of activation in right-sided olfactory eloquent centers, in right-handed participants [3]. This indicated either right-sided olfactory lateralization (irrespective of handedness) or ipsilateral olfactory hemispheric dominance with respect to handedness (where the right cerebral hemisphere is dominant in right-handed people). This view has been challenged, with studies (e.g. [4]) showing no difference in odor thresholds in relation to handedness, and research [5] showing no influence of handedness, but a general right-nostril advantage in odor discrimination.

The olfactory sulcus (OS) demarcates the division between the medial orbitofrontal gyrus and the rectus gyrus. It can be easily measured using structural magnetic resonance imaging (MRI). Previous studies have demonstrated reduced OS depth in patients with congenital anosmia [6, 7]. Hummel et al. [8] showed that olfactory function correlates significantly with left-sided depth of the OS whereas no such correlation was seen for the right side. Still, OS depth was found to be significantly deeper on the right compared to the left side. Whether OS depth and correlation with olfactory function is affected by handedness is unclear. Hence, we aimed to determine whether OS depth is different in right- or left-handed participants.

\section{Methods and Materials}

Forty-two healthy volunteers with a mean age of 24.1 years (range 18-45 years) were included. Ten of 23 right-handed and 10 of 19 left-handed participants were men (Table 1). None of the subjects was ambidextrous or had a history of handedness re-education. The study was performed in accordance with the Declaration of Helsinki, and all subjects provided informed written consent prior to inclusion. The study protocol was approved by the Ethics Committee at the TU Dresden (EK41022009).

MRI Protocol

MRI was performed using a 1.5-T scanner (SONATA-MR; Siemens, Erlangen, Germany). T1-weighted images were acquired using a 3D IR/GR sequence (TR: 2,180 ms/TE: $3.39 \mathrm{~ms}$ ).

Sniffin' Sticks Test

Birhinal cued odor identification was performed using the Sniffin' Sticks, which is a validated and reliable psychophysical test. The testing procedure has been extensively described elsewhere [9]. In brief, 16 odor stimuli are presented to the participant using a 4-alternate forced choice paradigm. Higher scores (maximum 16) indicate better olfactory function.

Sulcus Measurement

The depth of the OS (expressed in millimeters) was measured using 3D Slicer 4.10.1 (Isomics Inc., Kitware Inc. and GE Global Research) [10] for Windows. We used coronal sections. Measurements were 
Zang et al.: Olfactory Sulcus and Handedness

Table 1. Main statistics of the subjects

\begin{tabular}{|c|c|c|c|c|c|c|}
\hline & Mean & SD & Median & IQR & Q1 & Q3 \\
\hline \multicolumn{7}{|c|}{ Right-handers ( $n=23 ; 14$ women, 9 men) } \\
\hline Age, years ${ }^{a}$ & & & 23 & 3 & 22 & 25 \\
\hline \multicolumn{7}{|l|}{ OS depth, mm } \\
\hline Right OS depth ${ }^{\mathrm{b}}$ & 11.28 & 2.02 & & & & \\
\hline Left OS depth ${ }^{b}$ & 10.60 & 1.58 & & & & \\
\hline Identification scores $^{\mathrm{a}}$ & & & 15 & 1 & 14 & 15 \\
\hline Self-assessment ${ }^{\mathrm{a}}$ & & & 0 & 1 & 0 & 1 \\
\hline \multicolumn{7}{|c|}{ Left-handers ( $n=19 ; 7$ women, 12 men) } \\
\hline Age, years ${ }^{\mathrm{a}}$ & & & 24 & 4 & 21 & 25 \\
\hline \multicolumn{7}{|l|}{ OS depth, mm } \\
\hline Right OS depth ${ }^{b}$ & 11.26 & 1.84 & & & & \\
\hline Left OS depth ${ }^{b}$ & 10.98 & 1.45 & & & & \\
\hline Identification scores ${ }^{a}$ & & & 14 & 2 & 13 & 14.5 \\
\hline Self-assessment ${ }^{\mathrm{a}}$ & & & 0 & 1 & 0 & 1 \\
\hline
\end{tabular}

a The data do not obey the normal distribution after SK Normality test. ${ }^{\mathrm{b}}$ The data obey the normal distribution after SK Normality test.

performed in the plane of the posterior tangent through the eyeball, as per previous studies [11-13]. At the level of that plane, the depth of the OS was measured by drawing a straight line beginning with the deepest point of the sulcus and ending at the tangent connecting the top surfaces of the gyrus rectus and medial orbital gyrus [14] (Fig. 1).

\section{Self-Assessment}

All participants rated their olfactory sensitivity as "excellent" (score: +3), "very good," "good," "normal" (score: 0), "less than normal," "bad," "very bad," "no sense of smell” (score: -4 ) before olfactory testing was performed, with scores ranging from 3 to -4 .

\section{Data Analysis}

Results were analyzed with SPSS 25 (SPSS Inc., Chicago, IL, USA). The Shapiro-Wilk test was used to assess normal distribution of the data, and parametric or nonparametric tests were used accordingly. The depth of right and left OS in right- and left-handed participants were analyzed using paired samples $t$ tests. Mann-Whitney U tests were used to analyze differences in odor identification scores, lateralization test scores and self-ratings of olfactory function between right- and left-handed participants. The relationship between depth of right and left OS, odor identification scores, lateralization test scores and self-ratings of olfactory function and right and left handedness were tested using Spearman's correlation coefficient. The $\alpha$ level was set at 0.05 .

\section{Results}

\section{Depth of Right and Left OS in Right- and Left-Handers}

There were no significant differences between right-handed and left-handed participants on right/left OS depth $(p>0.05)$. When analyzing the subgroups, there was also no significant difference between right and left OS depth in the right-handed cohort or the left-handed cohort ( $p>0.05$ ). However, across all subjects (right- + left-handed) the mean depth of the right OS was significantly larger than that of the left OS $\left(t_{41}=-1.98, p=0.03\right)$ (Fig. 2). 
Fig. 1. The figure shows how to measure the olfactory sulcus (OS) depth in the plane of the posterior tangent through the eyeball. The first step is to draw a tangent to the top surfaces of the gyrus rectus and medial orbital gyrus. Then a line is drawn that connects the tangent to the deepest point of the OS. The length of this line is used as an estimate of the depth of the OS.

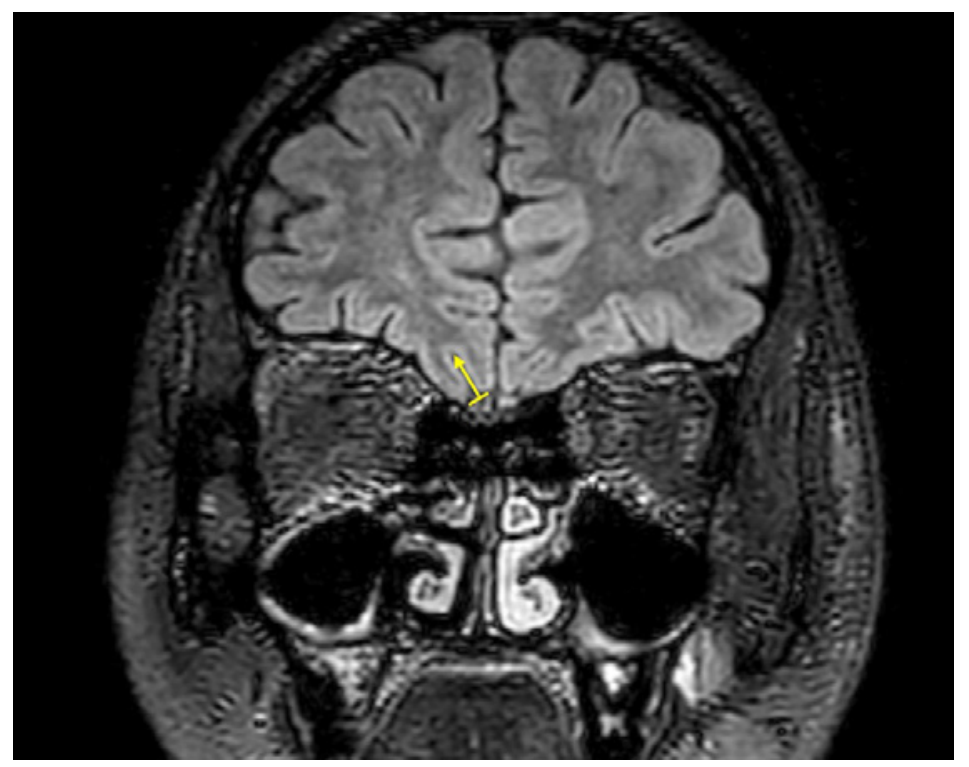

Fig. 2. Comparison between right and left OS depth across all participants (right and left handedness). ${ }^{*} p<0.05$ : mean significant difference.

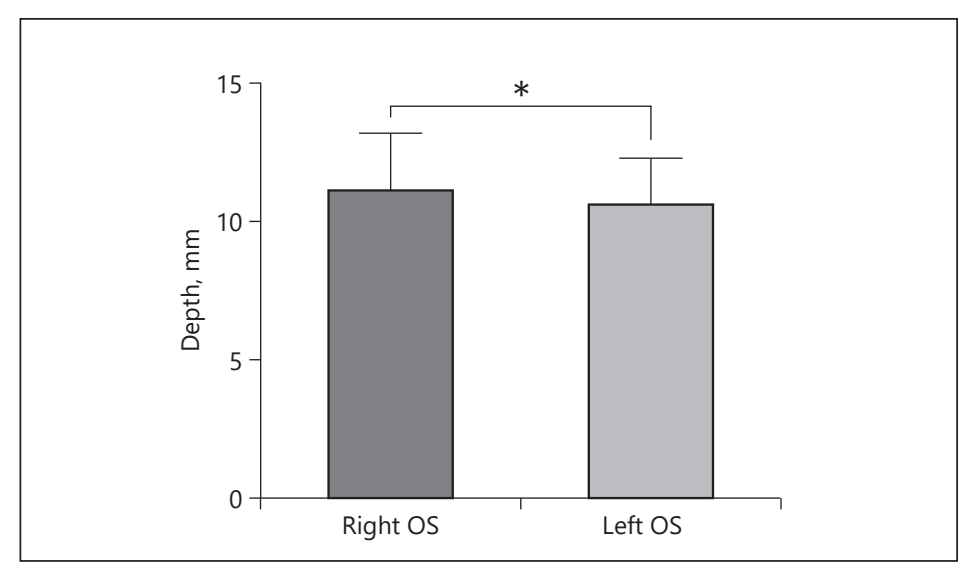

\section{Sniffin' Sticks Test}

There were no significant differences in birhinal odor identification score $(z=-1.67, p=$ $0.10)$ or self-rated olfactory function $(z=-0.58, p=0.56)$ between right- and left-handed participants. Further, there was no significant correlation between the identification scores of right-handed participants and right or left OS depth $(p>0.05)$. Similarly, the identification scores of left-handers did not correlate with right or left OS depth $(p>0.05)$. There was also no significant correlation between identification scores and right or left OS depth when both left- and right-handed cohorts were combined ( $p>0.05)$.

\section{Discussion}

The present study was designed to investigate whether the right or left OS depth is different in right- or left-handed participants.

We found that handedness exhibits no significant correlation with the OS depth. However, the current study confirmed that the right OS depth was significantly deeper than left OS depth across all participants. This result is in line with previous research which indicated 
deeper OS on the right side obtained in mixed right/left-handed cohorts [8]. The result seems to reflect lateralization of olfactory function, although this requires further research.

In studies on rat brains it was found that the total olfactory bulb volume and the outer striatum are larger on the right side. The authors speculated that the morphological asymmetry may reflect a difference in terms of functional asymmetrical organization of the olfactory pathways [15]. Some human brain anatomy studies on olfactory function lateralization reported greater deficits in patients with right temporal lesions in olfactory tasks, e.g., odor matching and odor recognition [16-18], which indicates from a clinical point of view that the right temporal lobe may play a different role in processing of olfactory information as compared with the left temporal lobe. Deficits in odor discrimination are more pronounced after damage to the right orbitofrontal cortex [5], and odor memory was impaired in patients with a right temporal lobectomy or right temporal-plus-frontal lesion, whereas patients with a left-sided lesion did not show significantly worse odor memory compared to healthy subjects [19]. Right hemispheric dominance for the processing of olfactory information was also shown in several other studies [20,21], although this is a matter of discussion [22].

Surprisingly, in the current study, we did neither find a significant correlation between birhinal olfactory function and right or left OS depth across all participants nor for right- and left-handed subgroups. This result is inconsistent with previous studies which indicated that olfactory function is significantly correlated with either right- [11] or left-sided OS depth [8]. The reason that we obtained these negative results may due to the olfactory measurements that were performed birhinally and not in a lateralized fashion. A different issue may relate to the odor identification test that has been used in this study. It may be hypothesized that odor identification is less sensitive to lateralization phenomena compared to odor discrimination which appears to tap into different domains of the processing of olfactory information [23].

\section{Conclusion}

Across all subjects the right OS was deeper compared to the left OS, which indicates right hemisphere dominance in odor perception. However, this was not related to handedness in the present study.

\section{Acknowledgment}

We would like to thank Marie Gottschlich for her help with data acquisition.

\section{Statement of Ethics}

All procedures performed in studies involving human participants were in accordance with the ethical standards of the institutional and/or national research committee and with the 1964 Helsinki Declaration and its later amendments or comparable ethical standards. The study was approved by the Ethics committee of the Medical Faculty of the Technical University of Dresden (EK41022009). All subjects provided informed written consent prior to inclusion. This article does not contain any studies with animals performed by any of the authors.

\section{Disclosure Statement}

The authors have no conflicts of interest to declare. 
Zang et al.: Olfactory Sulcus and Handedness

\section{Funding Sources}

This research received financial support by the Deutsche Forschungsgemeinschaft to T.H. (DFG HU411/18-1).

\section{Author Contributions}

Y. Zang, K.L. Whitcroft, C. Glöckler and T. Hummel contributed to the analysis of the results and to the writing of the manuscript, T. Hummel contributed to the design and implementation of the research.

\section{References}

1 Sun T, Walsh CA. Molecular approaches to brain asymmetry and handedness. Nat Rev Neurosci. 2006 Aug; 7(8):655-62.

2 Buck L, Bargmann C. Smell and taste: the chemical senses. In: Kandel E, Schwartz J, Siegelbaum SA, Hudspeth AJ, editors. Principles of neuroscience. 5th ed. New York: McGraw-Hill Medical; 2000. p. 712-42.

3 Li W, Lopez L, Osher J, Howard JD, Parrish TB, Gottfried JA. Right orbitofrontal cortex mediates conscious olfactory perception. Psychol Sci. 2010 Oct;21(10):1454-63.

4 Hummel T, Mohammadian P, Kobal G. Handedness is a determining factor in lateralized olfactory discrimination. Chem Senses. 1998 Oct;23(5):541-4.

5 Zatorre RJ, Jones-Gotman M. Human olfactory discrimination after unilateral frontal or temporal lobectomy. Brain. 1991 Feb;114 Pt 1A:71-84.

6 Huart C, Meusel T, Gerber J, Duprez T, Rombaux P, Hummel T. The depth of the olfactory sulcus is an indicator of congenital anosmia. AJNR Am J Neuroradiol. 2011 Nov-Dec;32(10):1911-4.

7 Abolmaali ND, Hietschold V, Vogl TJ, Hüttenbrink KB, Hummel T. MR evaluation in patients with isolated anosmia since birth or early childhood. AJNR Am J Neuroradiol. 2002 Jan;23(1):157-64.

8 Hummel T, Damm M, Vent J, Schmidt M, Theissen P, Larsson M, et al. Depth of olfactory sulcus and olfactory function. Brain Res. 2003 Jun;975(1-2):85-9.

9 Whitcroft KL, Cuevas M, Haehner A, Hummel T. Patterns of olfactory impairment reflect underlying disease etiology. Laryngoscope. 2017 Feb;127(2):291-5.

10 Fedorov A, Beichel R, Kalpathy-Cramer J, Finet J, Fillion-Robin JC, Pujol S, et al. 3D Slicer as an image computing platform for the Quantitative Imaging Network. Magn Reson Imaging. 2012 Nov;30(9):1323-41.

11 Hummel T, Urbig A, Huart C, Duprez T, Rombaux P. Volume of olfactory bulb and depth of olfactory sulcus in 378 consecutive patients with olfactory loss. J Neurol. 2015;262(4):1046-51.

12 Rombaux P, Duprez T, Hummel T. Olfactory bulb volume in the clinical assessment of olfactory dysfunction. Rhinology. 2009 Mar;47(1):3-9.

13 Kim JY, Lee WY, Chung EJ, Dhong HJ. Analysis of olfactory function and the depth of olfactory sulcus in patients with Parkinson's disease. Mov Disord. 2007 Aug;22(11):1563-6.

14 Rombaux P, Grandin C, Duprez TJB-E. How to measure olfactory bulb volume and olfactory sulcus depth? B-ENT. 2009 Jan;5:53-60.

15 Heine 0, Galaburda AM. Olfactory asymmetry in the rat brain. Exp Neurol.1986 Feb;91(2):392-8.

16 Abraham A, Mathai KV. The effect of right temporal lobe lesions on matching of smells. Neuropsychologia. 1983;21(3):277-81.

17 Jones-Gotman M. Contribution of the right temporal lobe to odor memory. Epilepsia. 1998;29:661.

18 Rausch R, Serafetinides EA, Crandall PH. Olfactory memory in patients with anterior temporal lobectomy. Cortex. 1977 Dec;13(4):445-52.

19 Jones-Gotman M, Zatorre RJ. Odor recognition memory in humans: role of right temporal and orbitofrontal regions. Brain Cogn. 1993 Jul;22(2):182-98.

20 Levy LM, Henkin RI, Hutter A, Lin CS, Martins D, Schellinger D. Functional MRI of human olfaction. J Comput Assist Tomogr. 1997 Nov-Dec;21(6):849-56.

21 Zatorre R, Jones-Gotman M, Evans A, Meyer E. Functional localization of human olfactory cortex with positron emission tomography. Nature. 1992;360:339-40.

22 Doty RL, Bromley SM, Moberg PJ, Hummel T. Laterality in human nasal chemoreception. Adv Psychol. 1997; 123:497-542.

23 Lötsch J, Reichmann H, Hummel T. Different odor tests contribute differently to the evaluation of olfactory loss. Chem Senses. 2008 Jan;33(1):17-21. 